\title{
OSCILLATION OF NONLINEAR SYSTEMS OF MATRIX DIFFERENTIAL EQUATIONS
}

\author{
A. G. KARTSATOS
}

ABSTRACT. For systems of matrix equations of the form

$$
U^{\prime}=A(t, U, V) V, \quad V^{\prime}=-B(t, U, V)
$$

it is shown here that the oscillation problem can be reduced to the corresponding problem of "associated" (in some sense) scalar equations for which there exist numerous results. Furthermore, it is also shown that many of the existing results concerning the equation

$$
\left(A(t) U^{\prime}\right)^{\prime}+B\left(t, U, U^{\prime}\right) U=0
$$

can be considerably improved by application of the above method.

This paper concerns itself with the oscillation of systems of matrix differential equations

$$
U^{\prime}=A(t, U, V) V, \quad V^{\prime}=-B(t, U, V)
$$

where $U=\left(u_{i j}\right), \quad V=\left(v_{i j}\right), A=\left(a_{i j}\right)$, and $B=\left(b_{i j}\right)$ are real $n \times n$ matrices. By $F(t, U, V)=f_{i j}(t, U, V)$ we mean $f_{i j}$ $=f_{i j}\left(t, u_{11}, \cdots, u_{n n}, v_{11}, \cdots, v_{n n}\right)$. The functions $a_{i j}, b_{i j}$ will be assumed continuous on $I \times R^{2 n}$, where $I=\left[t_{0},+\infty\right)$. The matrices $A(t, U, V), B(t, U, V) U^{-1}$ are symmetric for every pair $\{U, V\}$ such that det $U \neq 0$, and, moreover, $A(t, U, V)$ is positive definite for every $t \in I$ and every pair $\{U, V\}$. By a solution of $\left(^{*}\right)$ we mean here any pair $\{U, V\}$ of differentiable matrices which are defined on an infinite interval $I_{U, V} \subseteq I$ (depending on the particular pair) and satisfy $\left(^{*}\right)$ on this interval. Extending the concept of a "prepared solution" (from the linear case), we shall say that $\{U, V\}$ is a prepared solution of $\left(^{*}\right)$ if it is a solution such that:

$$
U^{*}(t) V(t)=V^{*}(t) U(t), \quad t \in I_{U, V},
$$

( $R^{*}$ denotes the transpose of the matrix $R$ ).

It is true that in the linear case we do not "lose" much by considering only prepared solutions, for, there, every solution is a linear combination of prepared ones (Barrett [1]). Here we shall also be concerned only with the prepared solutions of $\left({ }^{*}\right)$. The system $\left({ }^{*}\right)$ is

Received by the editors August 31, 1970 and, in revised form, December 4, 1970. AMS 1969 subject classifications. Primary 3442, 3445, 3490.

Key words and phrases. Nonlinear system of matrix differential equations, oscillatory system, oscillatory linear equation.

Copyright @ 1971, American Mathematical Society 
said to be oscillatory, if for every prepared solution $\{U, V\}$ of $\left(^{*}\right)$, the det $U$ has an unbounded sequence of zeros in $I_{U, V}$. Recent results concerning the existence of solutions to linear equations allow us to assume the existence of prepared solutions of $\left(^{*}\right)$, and this we do throughout the paper without further mention.

The following known theorems concern the differential equation

$$
\left(A_{0}(t) U^{\prime}\right)^{\prime}+B\left(t, U, U^{\prime}\right) U=0 .
$$

Theorem 1 (Howard [3]). Suppose that $A_{0}(t)$ is positive definite, $B \equiv B(t)$, and, moreover,

(i) there exists a positive definite scalar matrix $G=g(t) I$ such that

(ii) $\int_{i_{0}}^{+\infty} g^{-1}(t) d t=+\infty$,

(iii) $\lim _{t \rightarrow+\infty}\left(\inf _{x} x^{*} K(t) x\right)=+\infty$ where $x$ is a column vector of unit length,

$$
K(t)=\int_{t_{0}}^{t}\left[G(s) B(s)-(1 / 4) A_{0}(s)\left(G^{\prime}(s)\right)^{2} G^{-1}(s)\right] d s+\frac{1}{2} A_{0}(t) G^{\prime}(t),
$$

and

(iv) $\left(G(t) A_{0}(t)\right)^{-1} \geqq q(t) I$ where $q>0$ and $\int_{t_{0}}^{+\infty} q(t) d t=+\infty$.

Then $(* *)$ is oscillatory.

Theorem 2 (Tomastik [7]). Suppose that $A_{0}(t) \equiv I, B$ is positive definite and

$$
\lambda_{1}\left[\int_{t_{0}}^{+\infty} B\left(t, A(t), A^{\prime}(t)\right) d t\right]=+\infty
$$

for every differentiable matrix $A(t)$ such that $\operatorname{det} A(t) \neq 0$, and $\lambda_{n}\left[A^{*}(t) A(t)\right] \geqq \epsilon>0$. Then $\left({ }^{*}\right)$ is oscillatory.

Theorem 3 (Tomastik [7]). If $B$ is as in Theorem 2 and $A_{0}(t)$ $\equiv r(t) I$, where $r(t)$ is a positive scalar function and $\int_{t_{0}}^{+\infty} r^{-1}(t) d t=+\infty$, then $(* *)$ is oscillatory.

Theorem 4 (Swanson [6]). If $A, B$ are positive definite and $A$ is bounded above, and $\int_{t_{0}}^{+\infty} \lambda_{1}\left[B\left(t, A(t), A^{\prime}(t)\right)\right] d t=+\infty$ for every differentiable matrix $A$ such that $\operatorname{det} A(t) \neq 0$, for all large $t$, then $\left(^{* *}\right)$ is oscillatory.

In the above theorems, a prepared solution for $(* *)$ is a solution $U(t), t \in I_{U}$, such that $U^{*}(t) A_{0}(t) U^{\prime}(t)=U^{* \prime}(t) A_{0}(t) U(t)$, and $\lambda_{1}[A]$, $\lambda_{2}[A], \cdots, \lambda_{n}[A]$ are the eigenvalues of a symmetric matrix $A$ ordered in the following way: $\lambda_{1}[A] \geqq \lambda_{2}[A] \geqq \cdots \geqq \lambda_{n}[A]$.

Our aim here is to show that for large classes of systems of the 
form $\left({ }^{*}\right)$, the problem of oscillation can be reduced to that of corresponding (in some sense) scalar equations for which there is an abundance of oscillation criteria. At the same time we show how the above theorems can be improved considerably by means of this method. Before we state our main result, we show the validity of some properties of nonoscillatory solutions of $\left({ }^{*}\right)$ which do hold in the case of the equation $\left(^{* *}\right)$. In fact, if $\{U, V\}$ is a prepared solution of $\left(^{*}\right)$ which is not oscillatory, then there exists an interval $I_{1}$ $=\left[t_{1},+\infty\right) \subseteq I_{U, V}$ such that $\operatorname{det} U(t) \neq 0, t \in I_{1}$. Consequently, $U^{-1}(t)$ exists on $I_{1}$ and, by the preparedness of $U, V$, we obtain

$$
S \equiv V U^{-1}=\left[U^{*}\right]^{-1} V^{*} U U^{-1}=\left[U^{*}\right]^{-1} V^{*}=\left[V U^{-1}\right]^{*}
$$

which proves that the matrix $S(t)$ is symmetric on $I_{1}$. Now, by differentiation of $S(t)$, we obtain

$$
S^{\prime}(t)=-S(t) A(t, U(t), V(t)) S(t)-B(t, U(t), V(t)) U^{-1}(t) .
$$

If, in addition, we assume that the matrix $B U^{-1}$ is positive definite, then (4) implies that $S^{\prime}(t)$ is a negative definite matrix, i.e. (as in Tomastik's Theorem 1 in [6]) $S(t), V(t)$ are nonsingular on some interval $I_{2}=\left[t_{2},+\infty\right) \subseteq I_{1}$.

Our main result, which follows, extends a result of Barrett [1].

THEOREM 5. The equation (*) is oscillatory if for some $k(1 \leqq k \leqq n)$ and every pair of prepared differentiable matrices $U, V$ with $\operatorname{det} U \neq 0$, the equation

$$
\begin{aligned}
\left(a_{k k}^{-1}(t, U(t), V(t)) x^{\prime}\right)^{\prime}+\left[B(t, U(t), V(t)) U^{-1}(t)\right]_{k k} x & =0, \\
t & \in\left[t_{0},+\infty\right),
\end{aligned}
$$

is oscillatory, where the matrix $A_{k}(t, U, V)$ (which equals $A(t, U, V)$ with the kk-entry replaced by zero) is positive definite for any $t \in\left[t_{0},+\infty\right)$ and any $U, V$ as above.

Proof. Assume the existence of a nonoscillatory solution $\{U(t), V(t)\}, t \in I_{U, V}$, of $\left(^{*}\right)$ for which we have $\operatorname{det} U(t) \neq 0, t \in I_{1}$ $=\left[t_{1},+\infty\right) \subseteq I_{U, V}$. Then from (4) we obtain

$$
\begin{aligned}
S^{\prime}(t)= & -S(t)\left[A(t, U(t), V(t))-A_{k}(t, U(t), V(t))\right] S(t) \\
& -\left[S(t) A_{k}(t, U(t), V(t)) S(t)+B(t, U(t), V(t)) U^{-1}(t)\right]
\end{aligned}
$$

which leads to the Riccati equation

$$
S_{k k}^{\prime}(t)+A_{k k}(t) S_{k k}^{2}(t)+Q_{k k}(t)=0, \quad t \in I_{1},
$$


where $A_{k k}(t) \equiv a_{k k}(t, U(t), V(t))$ and $Q_{k k}(t) \geqq\left[B(t, U(t), V(t)) U^{-1}(t)\right]_{k k}$ because all the diagonal elements of $S A_{k} S$ are nonnegative (recall that $S$ is symmetric).

Now, by use of the substitution $x(t)=\exp \left[\int_{t_{1}}^{t} S_{k k}(s) d s\right]$, the equation (6) is transformed into

$$
\left(A_{k k}^{-1}(t) x^{\prime}\right)^{\prime}+Q_{k k} x=0
$$

and this is oscillatory by hypothesis. In view of the fact that $x(t)$ is a continuous positive solution of (7), the contradiction follows. Thus $\left.{ }^{*}\right)$ is oscillatory.

It is clear that in the above theorem we made use of the positive semidefiniteness of the matrix $A_{k}$ only in order to carry out the step from (5) to (6). It is understood that the conclusion would remain valid if one could obtain the desired contradiction directly from the equation

$$
S_{k k}^{\prime}(t)+[S(t) A(t, U(t), V(t)) S(t)]_{k k}+\left[B(t, U(t), V(t)) U^{-1}(t)\right]_{k k}=0
$$

and this reduces the problem to the study of the term in the middle.

We also note that the result in Theorem 5 can be proved if we suppose that $(E)$ is oscillatory for any pair of differentiable matrices $U, V$ such that $U^{*} V=V^{*} U=$ positive definite, provided that $\lambda_{i}\left[\int_{t_{0}}^{+\infty} A(t, U(t), V(t)) d t\right]=+\infty$ and $B U^{-1}=$ positive definite for matrices as in the theorem. In fact, in this case (4) implies

$$
\begin{aligned}
S^{-1}(t)= & S^{-1}\left(t_{1}\right)+\int_{t_{1}}^{t} S^{-1} B(s, U(s), V(s)) S^{-1}(s) d s \\
& +\int_{t_{1}}^{t} A(s, U(s), V(s)) d s
\end{aligned}
$$

from which follows (as in [7]) the positive definiteness of $S(t)$. Moreover, $U^{*} V=U^{*} S U$. This assumption is equivalent to $\lambda_{n}\left[A^{*}(t) A_{0}(t)\right]$ $\geqq \epsilon>0$ if the matrix $A_{0}$ is as in Theorem 3 .

Since the extension of results of the type of Theorems 1-4 to the system $\left({ }^{*}\right)$ is quite straightforward, we prefer to show, instead, that the procedure followed here leads to better results.

Consider first Theorem 1. In this case we have $A \equiv A_{0}^{-1}(t)$ and $B \equiv B_{1}(t) U$. Here we can merely suppose that there exists a function $g(t)>0, \int_{t_{0}}^{+\infty} g^{-1}(t)=+\infty$, such that

$$
\lim _{t \rightarrow+\infty} \int_{t_{0}}^{t}\left[g(s) b_{k k}^{1}(s)-\frac{1}{4} \bar{A}_{k k}^{-1}\left(g^{\prime}(s)\right)^{2} g^{-1}(s)\right] d s+\frac{1}{2} \bar{A}_{k k}^{-1}(t) g^{\prime}(t)=+\infty
$$


and

$$
\left[\bar{A}_{k k}^{-1}(t) g(t)\right]^{-1} \geqq q(t)>0 \text { with } \int_{t_{0}}^{+\infty} q(t) d t=+\infty,
$$

and the result follows as in Howard's theorem in [2].

As regards Theorem 2, we can replace (1) by the following considerably weaker assumption: for some $k(1 \leqq k \leqq n)$

$$
\int_{t_{0}}^{+\infty} t^{1-\epsilon} B_{k k}\left(t, A(t), A^{\prime}(t)\right) d t=+\infty \quad(0<\epsilon<1)
$$

for matrices as in Theorem 2, and the conclusion follows from a theorem of Mikusiński in [4].

Theorem 3 can be improved if we assume the existence of a function $g>0, \int_{t_{0}}^{+\infty} g^{-1}(t) d t=+\infty$, and

$$
\int_{t_{0}}^{+\infty} g(t)\left[B_{k k}\left(t, A(t), A^{\prime}(t)\right)-\frac{1}{4} r(t)\left(g^{\prime}(t)\right)^{2} g^{-2}(t)\right] d t=+\infty
$$

for every matrix $A$ as in Theorem 3. This result follows from Opial's theorem in [5]. In the case $g(t) \equiv 1$, we obtain Tomastik's theorem.

Finally, it is evident that we can obtain a result similar to that of Theorem 4 without requiring that the matrix $A_{0}$ be bounded above, but satisfying the assumptions of our theorem.

The author wishes to thank the referee for some helpful suggestions.

\section{REFERENCES}

1. J. H. Barrett, Matrix systems of second order differential equations, Portugal. Math. 14 (1956), 79-89. MR 18, 211.

2. H. C. Howard, Oscillation and nonoscillation criteria for $y^{\prime \prime}(x)+f(y(x)) p(x)=0$, Pacific J. Math. 12 (1962), 243-251. MR 26 \#2685.

3. - Oscillation criteria for matrix differential equations, Canad. J. Math. 19 (1967), 184-199. MR 35 \#3126.

4. J. G. Mikusiński, On Fite's oscillation theorems, Colloq. Math. 2 (1949), 34-39. MR $12,827$.

5. Z. Opial, Sur une critère d'oscillation des intégrales de l'équation différentielle $\left(Q(t) x^{\prime}\right)^{\prime}+f(t) x=0$, Ann. Polon. Math. 6 (1959/60), 99-104. MR 21 \#3627.

6. C. A. Swanson, Oscillation criteria for nonlinear matrix differential inequalities, Proc. Amer. Math. Soc. 24 (1970), 824-827.

7. E. C. Tomastik, Oscillation of nonlinear matrix differential equations of second order, Proc. Amer. Math. Soc. 19 (1968), 1427-1431. MR 38 \#372.

University of Athens, Athens (143), Greece 